\title{
Conformation and drift of a telechelic chain in porous media
}

\author{
Aniket Bhattacharya \\ Department of Physics, 4000 Central Florida Boulevard, University of Central Florida, Orlando, \\ FL 32816-2385, USA \\ E-mail: aniket@physics.ucf.edu
}

Received 25 August 2004

Published 22 October 2004

Online at stacks.iop.org/JPhysCM/16/S5203

doi:10.1088/0953-8984/16/44/017

\begin{abstract}
The conformation and drift properties of a telechelic chain moving through a porous host of randomly distributed static obstacles are studied using Brownian dynamics simulation for a Grest-Kremer bead-spring model of polymer. Static chain conformations exhibit non-monotonic behaviour as a function of the impurity density $\rho_{\text {imp }}$ at zero bias. The chain initially shrinks due to an entropic barrier as the density of the obstacles $\rho_{\mathrm{imp}}$ is increased. In the presence of the obstacles it requires only a tiny bias for a long chain to be mostly stretched. We then study the drift property of the chain as a function of the bias and impurity density. The drift velocity of the chain saturates beyond a threshold bias $F_{x}^{\text {crit }}$. This observation can be useful in designing DNA sequencing methods based on electrophoretic mobility.
\end{abstract}

(Some figures in this article are in colour only in the electronic version)

\section{Introduction}

The transport of polymers through nanochannels and gels [1, 2] is an active field of research. A deeper understanding of the mechanism of transport and chain mobility will lead to useful applications in the detection of sequences in DNA/RNA. A telechelic ionomer is a polymer which carries charges either in one or both ends. This is a special case of associative polymers which form thermoreversible gels [3]. We consider a telechelic chain with charge at one end. The chain can be dragged with an applied electric field. We ask how the static conformations are changed in the presence of randomly distributed obstacles. We then extend these calculations to include a bias, and study how the connectivity and flexibility influences the mobility of the chain.

The problem is a special case of a much broader field: namely, the behaviour of a polymer chain in random media [1,2]. At a fundamental level the study of a polymer chain in 
disordered media stands as a testing ground for the extension and applicability of the theory of reptation $[4,5]$. At the same time the flow property of a polymer chain can be immensely useful in understanding many important processes, for example length dependent fractionation of DNA via gel electrophoresis, complex polymer mixtures, hazardous waste in sea, etc.

For a Gaussian chain, both the Monte Carlo (MC) [6] calculations and analytic calculations using the replica trick [7] predict that in the presence of quenched disorder the chain shrinks and the length dependence of the diffusion constant is different to what is predicted by the reptation theory $[4,5]$. The extension of these studies from a Gaussian chain to a self-avoiding chain adds another dimension to the problem. For a self-avoiding chain, the competition between the strength of the excluded volume interaction $(w)$ and that of the disorder $(v)$ yields very interesting conformational and localization properties [8-10]. For a polymer chain of length $N$ embedded in porous media characterized by the impurity density $\rho_{\text {imp }}$, the variational calculations using the replica trick [8] predict three different regimes for the polymer conformations: while for $w>v, R_{\mathrm{g}}^{2} \sim N^{1.2}$, for $w=v$, the chain executes ordinary random walk $R_{\mathrm{g}}^{2} \sim N$, and for high impurity densities $(v>w)$, the chain is localized with $R_{\mathrm{g}}^{2} \sim \rho_{\mathrm{imp}}^{2 / 3} N^{2 / 3}$.

The MC calculations by Muthukumar and Baumgartner $[9,10]$ are consistent with these results. In contrast, a second analytic calculation using optimal fluctuation theory in terms of a small parameter predicts [11] that for large impurity densities the conformational properties of a chain are still described by a self-avoiding random walk. When a polymer chain moves through a random medium it encounters cavities of different lengths. As the radius of gyration $\left\langle R_{\mathrm{g}}\right\rangle$ of the chain becomes comparable to or larger than the mean pore size it encounters entropic barriers as it passes through bottlenecks [10]. It has been argued [11] that replica calculations relying on a single variational parameter might not be adequate to describe a more complicated free energy landscape, justifying the need for further work to settle some of these issues. The transport properties of a polymer chain through porous media $[9,12]$ reveal some unsettled issues as well. Earlier MC calculations suggest [9] that chain dynamics in a dense system can be different from reptation. But a more recent MC investigation of diffusion of a polymer chain through a sea of frozen polymer chains by Yamakov and Milchev [12] indicates that reptation is still the dominant mode of polymer diffusion in random media.

The above discussions refer to the diffusive and conformational properties of polymers in random media. Motivated by practical problems, for example the electrophoresis of biomolecules under an external field, these studies of neutral polymers have also been extended to polyelectrolytes where the entire chain is charged and driven by an electric field [1]. A special case of this variety is a telechelic polymer chain where only a block in either end of the chain is charged, and the rest of the chain is neutral. The conformational and drift properties of these end-labelled chains are of significant practical importance and have been studied previously analytically, and by MC and molecular dynamics (MD) simulation methods [13-19]. In the absence of bias, estimates of both the transverse and the longitudinal size of a stretched polymer chain can be obtained using simple scaling arguments $[4,20]$. Analytic results using the optimal fluctuation method indicate that the transverse fluctuations of the centre of mass of a polymer chain are similar to that of a Brownian particle and exhibit diffusive behaviour [13]. These calculations also obtain the first order correction to the drift velocity due to impurities. A recent $\mathrm{MC}$ simulation indicates that in the presence of the impurities, these scaling properties are weakly violated [16]. The simulation data also show that there exists a critical value of the bias beyond which a 'jamming effect' is observed [16].

The purpose of this paper is to examine the properties of an end-labelled telechelic polymer chain where only the first monomer of the chain is influenced by an external force using 
a stochastic molecular dynamics simulation method [21-23], and to calculate the dynamic properties of the chain directly from the simulation as a function of bias and impurity density. There are some distinct advantages of carrying out MD, as opposed to MC in this particular problem. In MD, the drift velocity and other dynamic properties can be obtained directly from the simulation and do not require any assumptions. For large bias, the MC approach may suffer practical difficulties, specifically in implementing the Boltzmann factor $\exp (-\beta \Delta E)$, while in MD a larger bias requires a smaller integration time step which renders the calculations more expensive but feasible. We also study the conformational and the diffusive properties of the chain at zero bias. Previously we have reported some properties for shorter chains [19]. Here we report results for longer chains and study other interesting quantities not reported previously.

The salient features of our calculations are as follows. For the zero bias case, we find that the radius of gyration $\left\langle R_{\mathrm{g}}\right\rangle$ of the chain is a non-monotonic function of the impurity density ( $\left.\rho_{\text {imp }}\right)$ exhibiting a minimum. This trend was not reported in the previous MC calculations [9], but has been observed in more recent MC calculations for a slightly different nature of the quenched disorder [15]. This indicates that the result has more general validity for a variety of disordered media. We calculate the Flory exponent $v$ using a chain length from 8 to 128 for impurity density $0.1-0.3$ and find $v \simeq 0.6$, which implies that the chain is still described by a self-avoiding random walk (SAW) at these densities. Finally, we observe that the drift velocity of the chain exhibits a saturation as a function of the bias. It appears that the saturation bias is inversely proportional to the chain length $N$. This observation can provide useful information in electrophoresis and other related experiments where the filtering method depends of the mobility of the chains.

The model and numerical methods are discussed in the following section. In section 3 we present the static and the dynamic properties of the chain. In sections 3 and 4 we summarize the results and discuss ongoing and prospective future work.

\section{Model and numerical procedure}

The molecular dynamics method that we have implemented here is the same as the one previously employed by the author [22] and very similar to the method adopted by Grest and co-workers earlier [21]. To simulate a constant temperature ensemble, the monomers are coupled to a heat bath and the equations of motion read as

$$
\ddot{\vec{r}}_{i}=-\vec{\nabla} U_{i}-\Gamma \dot{\vec{r}}_{i}+\vec{W}_{i}(t),
$$

where $\Gamma$ is the monomer friction coefficient and $\vec{W}_{i}(t)$ which describes the random force of the heat bath acting on each monomer is a Gaussian white noise with zero mean satisfying the fluctuation-dissipation relation

$$
\left\langle\vec{W}_{i}(t) \cdot \vec{W}_{j}\left(t^{\prime}\right)\right\rangle=6 k_{\mathrm{B}} T \Gamma \delta_{i j} \delta\left(t-t^{\prime}\right) .
$$

The potential $U_{i}$ consists of two parts: $U_{\mathrm{LJ}}$, and $U_{\text {chain }}$. Here $U_{\mathrm{LJ}}$ is a Lennard-Jones (LJ) potential acting between any two pair of monomers:

$$
U_{\mathrm{LJ}}(r)=4 \epsilon\left[\left(\frac{\sigma}{r}\right)^{12}-\left(\frac{\sigma}{r}\right)^{6}-\left(\frac{\sigma}{r_{\mathrm{c}}}\right)^{12}+\left(\frac{\sigma}{r_{\mathrm{c}}}\right)^{6}\right] ; \quad r \leqslant r_{\mathrm{c}},
$$

where $r_{\mathrm{c}}$ is the cutoff distance beyond which the LJ interaction is set to be zero and $r=\left|\vec{r}_{i}-\vec{r}_{j}\right|$, with $\vec{r}_{i}, \vec{r}_{j}$ being the locations of the $i$ th and $j$ th monomers, respectively. The parameter $\epsilon$ is the LJ energy parameter and $\sigma$ is the $\mathrm{LJ}$ length parameter. The LJ interaction is a purely repulsive potential if the cutoff distance $r_{\mathrm{c}}=2^{1 / 6} \sigma$, but has an attractive part when $r_{\mathrm{c}}=2.5 \sigma$. 
$U_{\text {chain }}$ is the finite-extendable nonlinear elastic (FENE) (anharmonic spring) potential acting between pairs of successive monomers along a chain:

$$
U_{\text {chain }}(r)=-k \ln \left[1-\left(\frac{l}{l_{0}}\right)^{2}\right],
$$

in which $k$ is the energy parameter of the potential, $l$ is the distance between two neighbouring monomers of the same chain, and $l_{0}$ is a length parameter which describes the extension range between two successive monomers. We have chosen $k=30$ and $l_{0}=1.5$, which make chain crossing practically impossible [21]. We use reduced units throughout this study. The unit of time is $\sigma(m / \epsilon)^{1 / 2}$ and the unit of temperature is $\epsilon / k_{\mathrm{B}}$, where $k_{\mathrm{B}}$ is the Boltzmann constant. The interaction of the polymer chain with the randomly placed impurities is described by the repulsive part of the LJ interaction. A constant force is added to the $x$ component of the force on the first monomer to simulate a telechelic chain.

We have integrated the equations of motion following an accurate scheme developed by van Gunsteren and Berendsen [23] which uses a bivariate distribution of Gaussian random numbers for the stochastic forces [24]. A very fast Gaussian random number generator [25] and a link-cell-list $[26,27]$ for calculating the forces help to make the integration quite efficient. Time steps for most of the cases were chosen to be $(\Delta \tau)$ of 0.01 (in reduced units) which produced stable integration at temperatures of interest. For a large bias and long chain in the presence of a high density of scatterer, $\Delta \tau \sim 0.0025$ was necessary, which rendered the calculations for chain length 64 and 128 time-consuming. The results that are presented here needed the continuous operation of six Linux boxes for several months.

\section{Results}

We show results for chain lengths $N=8,16,32,64$, and 128 and for impurity densities $\rho_{\text {imp }}=0.1,0.2,0.3,0.4$ and 0.5 for several values of bias $F_{x}$. Typically disorder is averaged over 500-1000 initial configurations, which makes these computations quite time-consuming. The computation time for chain lengths 64 and 128 are very large and the data sets are less exhaustive compared to smaller chains. Unless explicitly shown, the error bars for the static quantities are very small and within the width of the symbols in the figures.

\subsection{Static properties of the chain: zero bias}

First we show the results for the zero bias case and compare our numerical results with those obtained from the analytic theories, as well as previous MC results [12]. Figure 1 shows the normalized radius of gyration $\left\langle\bar{R}_{\mathrm{g}}\right\rangle=\left\langle R_{\mathrm{g}}\left(\rho_{\mathrm{imp}}\right)\right\rangle /\left\langle R_{\mathrm{g}}\left(\rho_{\mathrm{imp}}=0\right)\right\rangle$ as a function of impurity density $\rho_{\text {imp }}$ for chain lengths $N=8,16,32,64$, and 128 , respectively. The ubiquitous feature is that the average size of the chain initially decreases to a minimum value with increasing impurity density. The position of these minima for different $\rho_{\text {imp }}$ is a function of chain length $N$ and shifts to a lower value for longer chains. A similar result was found by Yamakov and Milchev for a different system where the disorder was made of an initially relaxed and subsequently frozen network of polymer chains. Since all the interactions considered here are purely repulsive, the origin of the observed minimum is due to the entropic traps and barriers [10]. Imagine a situation where a chain is fully confined in a particular cavity connected to other neighbouring cavities through bottlenecks. In this case the chain under consideration encounters entropic barriers to wander in to the neighbouring cavities, as explained by Muthukumar and co-workers. Now imagine that the impurities' distances are scaled down without affecting the chain so that the average pore size becomes smaller. 


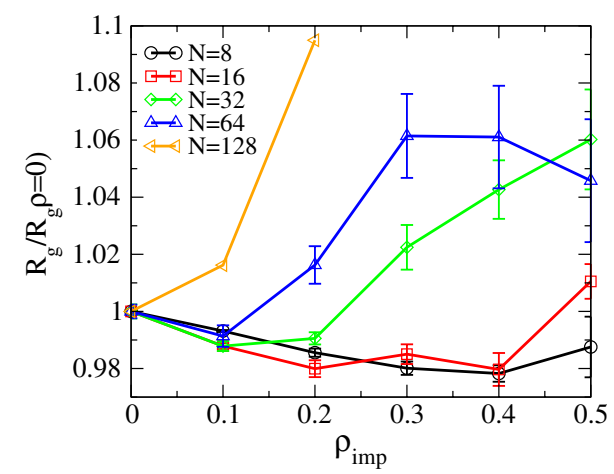

Figure 1. Normalized radius of gyration $\left\langle\bar{R}_{\mathrm{g}}\right\rangle$ at zero bias for different values of $\rho_{\text {imp }}$; circles, squares, diamonds, triangles and triangles (rotated) represent chain lengths $N=8,16,32,64$, and 128 , respectively. The curves through the points are guides to the eye. The error bars for $N=128$ are large and not shown.

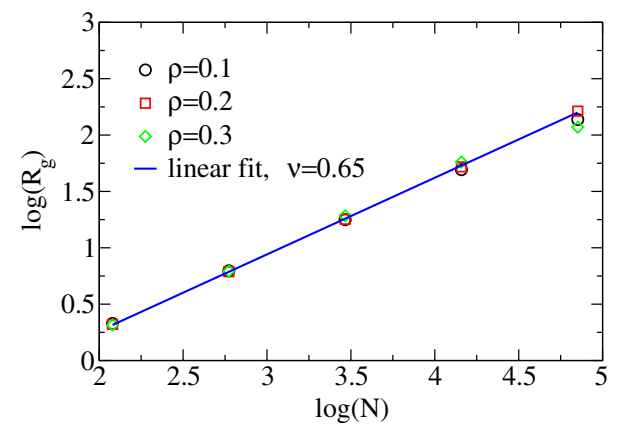

Figure 2. $R_{\mathrm{g}}$ as a function of chain length $N$ (log scale) for $\rho_{\text {imp }} 0.1$ (circles), 0.2 (squares), and 0.3 (diamonds). The slope of the curves is the same and gives $v \simeq 0.65$.

If the chain still could be accommodated in this particular pore it will have a smaller $\left\langle R_{\mathrm{g}}\right\rangle$. This continues until $\rho_{\text {imp }}$ reaches $\rho_{\mathrm{imp}}^{\min }$. For larger $\rho_{\mathrm{imp}}$, the average size of the cavities cannot accommodate the full chain and the chain stretches in between the cavities with an increased $\left\langle R_{\mathrm{g}}\right\rangle$, as alluded to in the work of Panyukov [11]. This qualitatively explains the origin of the minimum in figure 1 . For very long chains, this feature will be present only at extremely low impurity densities and, therefore, may not be observed in the simulation $(N=128)$. We have calculated the Flory exponent $v$ (see figure 2 ) for various values of the impurity density $\rho_{\text {imp }}$ by plotting $R_{\mathrm{g}}$ as a function of chain length $N$. At a certain obstacle density, $\rho_{\mathrm{imp}}$, the medium creates random channels, characterized by a 'persistent length' which exceeds the persistent length of the polymer chain and effectively rectifies it, thus leading to larger values of $v>0.6$.

\subsection{Static properties of the chain: nonzero bias}

The presence of a bias drastically alters the conformation properties of the chain. Two appropriate quantities of interests are: (i) the ratio $R=\left\langle R_{\mathrm{e}}^{2}\right\rangle /\left\langle R_{\mathrm{g}}^{2}\right\rangle$, where $\left\langle R_{\mathrm{g}}\right\rangle$ and $\left\langle R_{\mathrm{e}}\right\rangle$ represent the average radius of gyration and the end-to-end distance of the chain respectively, and (ii) the quantity $\Delta R_{x}^{y z}=\left(\left\langle R_{\mathrm{g}}\right\rangle_{x} /\left\langle R_{\mathrm{g}}\right\rangle\right)_{y z} \cdot R$ lies between 6 and 12 for a Gaussian and perfectly stretched chain; $\Delta R_{x}^{y z}$ will be unity in the absence of a bias. These two quantities will 

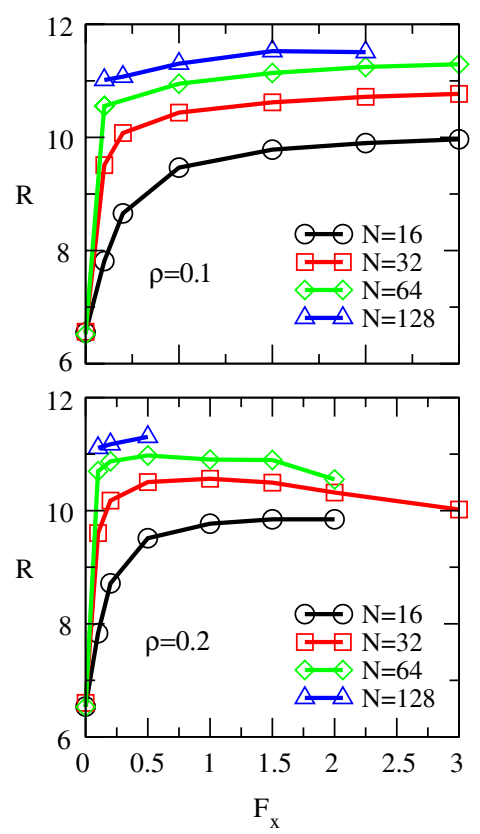

Figure 3. The ratio $R=\left\langle R_{\mathrm{e}}^{2}\right\rangle /\left\langle R_{\mathrm{g}}^{2}\right\rangle$ under bias for different impurity densities. Circles, squares, diamonds, and triangles represent chain lengths $N=16,32,64$, and 128, respectively. The lines through the points are guides to the eye.

be able to provide sufficient information to obtain a detailed picture of the chain conformation. For low densities of the obstacles and for shorter chains, $R$ increases with increasing bias without any sign of saturation. For high densities of the obstacles $R$ becomes very quickly insensitive to the bias. For moderate densities we find that $R$ exhibits a maximum and a subsequent weak decrease. We believe that this is a generic behaviour of the chain in a random medium in the presence of a bias. The peak position is a function both of the chain length and the bias. It is evident that the longer the chain is, the more sensitive it is to the external field. Even for a tiny bias the longer chain has a significantly larger value of $R$ compared to its value in the absence of the bias. We notice that longer chains $(N=64$ and 128) have a maximum value of $R \sim 11.5$. Therefore the chain is mostly stretched. For a given chain, an estimate of $F_{x}$ could be obtained from the dimensionless quantity $F_{x} R_{\mathrm{g}} / k_{\mathrm{B}} T$. We find that for low $\rho_{\text {imp }}$ the chain stretching reaches a saturation for $F_{x} R_{\mathrm{g}} k_{\mathrm{B}} T \sim 1$. Moreover, for $\rho_{\mathrm{imp}}=0.2$ the average pore spacing $r_{0}$ is $\sim 1.2$, while the radii of gyration for $N=16,32,64$, and 128 are $\sim 2.2,3.5,5.6$, and 9.2, respectively, which are larger than $r_{0}$. Therefore the ratio $\frac{r_{0}}{R_{\mathrm{g}}}$ dictates the minimum value of $F_{x}$ for which the chain is mostly stretched.

We get a more complete picture of the longitudinal and lateral dimensions of the chain from figure 4 . We notice that for moderate densities of the impurities and low bias, the chain is not only stretched along the direction of the bias but shrinks in the transverse direction. However, with increasing bias and impurity density, a saturation effect is observed, while with a further increase in the bias the effect is reversed. It is worth noticing that the effect of the impurity and the bias becomes more drastic with increasing chain length.

One may wonder to what extent the bonds are stretched when we apply a large bias. In figure 5 we show the average bond lengths as a function of the distance from the bead which is dragged. The stretching is within less than $1 \%$. But we notice that chain stretching becomes 

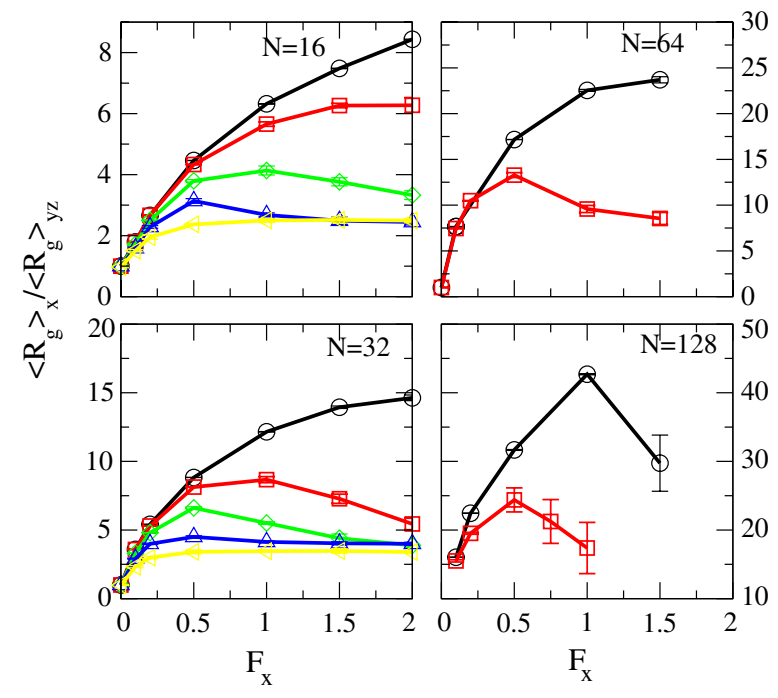

Figure 4. The ratio $\Delta R_{x}^{y z}$ under bias for different impurity densities. Circles, squares, diamonds, and triangles represent chain lengths $\rho_{\mathrm{imp}}=0.1,0.2,0.3$, and 0.4 , respectively. The curves through the points are guides to the eye.

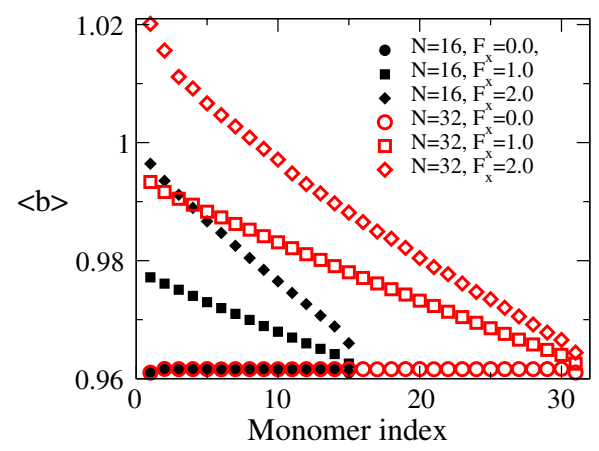

Figure 5. Average bond lengths between successive beads from one end of the chain. The bias is applied on the first bead.

more drastic for larger chains, rendering the calculations prohibitively large, especially for large bias.

\subsection{Dynamic properties of the chain}

Finally, we show the nonlinear dependence of the velocity of the centre of mass of the chain $V_{\mathrm{d}}$ on the bias $F_{x}$ for different $\rho_{\text {imp }}$ and $N$. For low $\rho_{\text {imp }}$, the velocity increases linearly with the increasing bias, as expected. In this limit $\left(F_{x} \rightarrow 0\right)$, we find the mobility of all the chains are roughly the same, as expected. As the bias is increased, the velocity exhibits a maximum for intermediate values of concentrations, as shown in figure 6. It appears from figure 6 that the critical bias $F_{x}^{\text {crit }} \sim \frac{1}{N}$ for $\rho_{\text {imp }}=0.2$. Occasionally, at large bias, the chain can overcome the entropic barrier and access regions of high entropic penalty from which it becomes difficult to get out, which explains its reduced drift velocity. If we compare figure 6 with 3 , we notice that the ratio for different chain lengths beyond $F_{x}^{\text {crit }}$ is significantly larger than 6 which indicates 


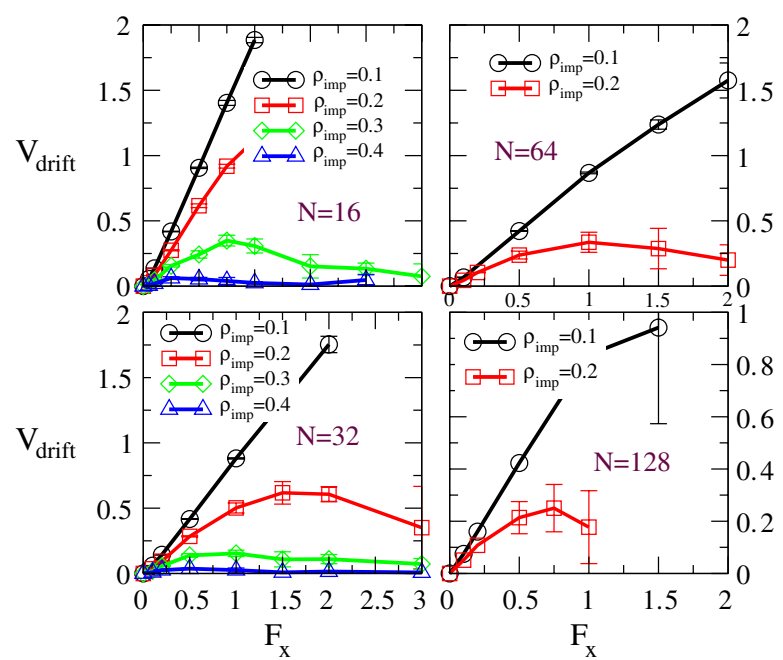

Figure 6. Drift velocity $V_{\text {drift }}$ of chains for different impurity density and bias. The drift velocity exhibits saturation.

that, for a large bias, the chain does not have sufficient time to meander its way around the obstacles and encounters the repulsive barrier of the obstacle as happens in a 'head-on' collision more frequently. The rest of the chain, being neutral, samples the available volume, which is consistent with the fact that the Flory exponent $v \geqslant 0.6$.

\section{Discussion}

In summary, we have investigated both the static and the dynamic properties of an end-labelled telechelic polymer chain in the presence of quenched random impurities and driven by an external field using a stochastic MD simulation method. Our numerical data show a minimum in $\left\langle R_{\mathrm{g}}\right\rangle$ with increasing density of randomness. The trend suggests that for very long chains this effect will disappear as the position of the minimum shifts towards $\rho_{\text {imp }} \rightarrow 0$ for longer chains. Further systematic runs with smaller densities and longer chains will settle this issue.

In the absence of a bias, our MD data reveal several important results. For impurity densities $\rho_{\text {imp }}=0.0-0.6$, our simulation results show that the effective Flory exponent $v_{\text {eff }} \geqslant 0.6$, which indicates that the conformation of the chain at high impurity density is still described by a self-avoiding random walk (albeit with the 'persistent length' of the medium, as proposed in a paper by Panyukov). This has also been observed previously in an MC calculation [15]. This is an important result, and is probably indicative of the limitations of the variational calculations using the replica trick, as it predicts otherwise.

The effect of the bias is more drastic for longer chains. Therefore it seems plausible that in the limit of very long chains and in the presence of a weak disorder, an extremely small but nonzero bias would be sufficient to stretch the chain completely. From our simulation data it appears that in the presence of disorder the simple scaling laws for the transverse and the longitudinal components are violated. A more refined scaling analysis would require an obvious additional variable $r_{0} /\left\langle R_{\mathrm{g}}\right\rangle$.

The observed maximum for the drift velocity as a function of the bias is consistent with the idea of jamming. Once the telechelic head gets stuck in a narrow channel among obstacles and the large bias does not let it get loose, it can take a very long time to escape out of 
that region, which would explain the observed reduction of the drift velocity at large bias. Note that the neutral tail of the polymer does not help the immobile head in this situation. It will be worthwhile to calculate the residence time for such an event as a function of the chain length, randomness and bias. At present we are extending our calculations to larger chain lengths and more numerous biases and impurity densities. In particular the intermediate density regime, where both the static and the dynamic properties exhibit extrema, are worth investigating in more detail. Our simulation does not take into account any hydrodynamic effects which may also play a role; however, one expects that at small free volume in the system hydrodynamic corrections will be small. The drift of the chain in various regular and periodic geometries may also reveals important differences when compared with the results reported in this paper. Furthermore, the MD approach will be very desirable to take into account the dynamic environment of the obstacles. Some of these issues are currently under investigation and will be reported in a separate publication.

\section{Acknowledgments}

The research reported here is supported in part by a grant from the National Science Foundation NIRT (ENG/ECS and CISE/EIA) under grant no. 0103587. The author thanks Andrey Milchev for many discussions and comments.

\section{References}

[1] Viovy J 2000 Rev. Mod. Phys. 72813

[2] Baumgartner A and Muthukumar M 1996 Advances in Chemical Physics vol XCIV, ed I Prigogine and S A Rice (New York: Wiley)

[3] Eisenberg A and Kim J 1998 Introduction to Ionomers (New York: Wiley)

[4] de Gennes P G 1979 Scaling Concepts in Polymer Physics (Ithaca, NY: Cornell University Press)

[5] Doi M and Edwards S F 1986 The Theory of Polymer Dynamics (Oxford: Oxford University Press)

[6] Baumgartner A and Muthukumar M 1987 J. Chem. Phys. 873082

[7] Edwards S F and Muthukumar M 1988 J. Chem. Phys. 892435

[8] Muthukumar M 1989 J. Chem. Phys. 904594

[9] Muthukumar M and Baumgartner A 1989 Macromolecules 221941

[10] Muthukumar M and Baumgartner A 1989 Macromolecules 221937

[11] Panyukov S V 1993 JETP 76631

[12] Yamakov V and Milchev A 1997 Phys. Rev. E 567043

[13] Stepanow S and Schultz M 1999 Europhys. Lett. 47643

[14] Wu S Y and Slater G W 1993 Macromolecules 261905

[15] Yamakov V and Milchev A 1997 Phys. Rev. E 551704

[16] Avramova K and Milchev A 2002 Eur. Phys. J. E 765

[17] Foo G M and Pandey R B 1995 Phys. Rev. E 515738

[18] Foo G M and Pandey R B 2000 J. Chem. Phys. 11210659

[19] Bhattacharya A and Milchev A 2002 Phys. Rev. E 66041806

[20] Pincus P 1976 Macromolecules 9386

[21] Kremer K and Grest G S 1990 J. Chem. Phys. 925057 See also articles by Kremer K and Grest G S 1995 Monte Carlo and Molecular Dynamics Simulations in Polymer Science ed K Binder (Oxford: Oxford University Press)

Grest G S and Murat M 1995 Monte Carlo and Molecular Dynamics Simulations in Polymer Science ed K Binder (Oxford: Oxford University Press)

[22] Bhattacharya A, Mahanti S D and Chakrabarti A 1998 Phys. Rev. Lett. 80333

[23] van Gunsteren W F and Berendsen H J C 1982 Mol. Phys. 45637

[24] Chandrasekhar S 1943 Rev. Mod. Phys. 151

[25] Toral R and Chakrabarti A 1993 Comput. Phys. Commun. 74327

[26] Grest G S, Dunweg B and Kremer K 1989 Comput. Phys. Commun. 55269

[27] Allen M and Tildesley D 1987 Computer Simulation of Liquids (Oxford: Clarendon) 